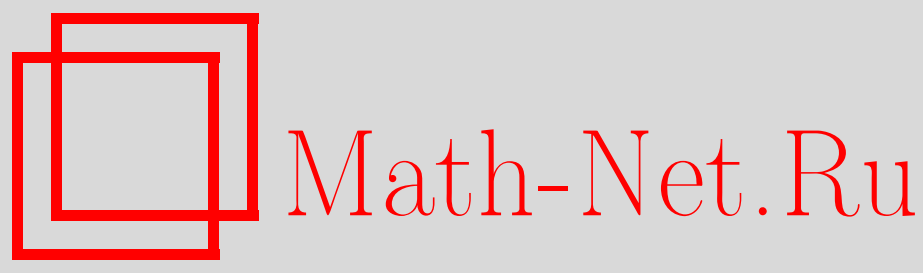

В. А. Гейлер, Д. А. Иванов, И. Ю. Попов, Аппроксимация точечных возмущений на римановом многообразии, ТМФ, 2009, том 158, номер 1, 49-57

DOI: https://doi.org/10.4213/tmf6298

Использование Общероссийского математического портала Math-Net.Ru подразумевает, что вы прочитали и согласны с пользовательским соглашением http://www . mathnet.ru/rus/agreement

Параметры загрузки :

IP : 18.234 .197 .8

26 апреля 2023 г., 15:05:18

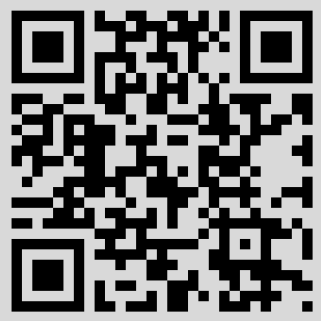




\title{
АППРОКСИМАЦИЯ ТОЧЕЧНЫХ ВОЗМУЩЕНИЙ НА РИМАНОВОМ МНОГООБРАЗИИ
}

\begin{abstract}
Показано, что гамильтониан точечного взаимодействия на римановом многообразии ограниченной геометрии может быть получен как предел (в смысле равномерной резольвентной сходимости) последовательности масштабно-преобразованных гамильтонианов с короткодействием.
\end{abstract}

Ключевые слова: риманово многообразие, точечное взаимодействие, аппроксимация.

\section{1. ВВЕДЕНИЕ}

Для изучения сложных систем в квантовой механике широко используются модели, описывающие взаимодействие заряженной частицы с потенциалом, сосредоточенным в одной точке (точечным потенциалом). Гамильтониан таких моделей формально представим в виде

$$
H=H^{0}+\lambda \delta_{q}(\cdot)
$$

где $H^{0}$ - квантово-механический гамильтониан свободной частицы, $\lambda$ - константа связи, а $\delta_{q}(\cdot)-\delta$-функция Дирака в точке $q$. В конкретных физических задачах свободный гамильтониан заменяется на соответствующий гамильтониан без сингулярного взаимодействия.

Начало изучению моделей такого вида было положено еще в 1931 г. [1]. Однако корректное математическое описание появилось лишь в работе [2] в рамках теории самосопряженных расширений симметрических операторов. Следует отметить, что подход теории расширений, обеспечивая строгий анализ, в то же время ставит вопрос о верификации модели, т.е. о выборе параметров расширений, обеспечивающих необходимое соответствие модели и реальной задачи. Это обоснование модели возможно путем аппроксимации точечных потенциалов гладкими. В рамках настоящей работы нас будет интересовать именно этот вопрос. Что касается моделей в $\mathbb{R}^{3}\left(\mathbb{R}^{2}\right)$,

* Мордовский государственный университет им. Н. П. Огарева, Саранск, Россия. E-mail: ivdmal@mail.ru

${ }^{\dagger}$ Санкт-Петербургский государственный университет информационных технологий, механики и оптики, Санкт-Петербург, Россия. E-mail: popov@mail.ifmo.ru 
то исчерпывающее описание процесса построения такой аппроксимации изложено, например, в монографии [3]. В нашей работе речь пойдет о более общих пространствах.

В связи с интенсивными исследованиями спектра примесей в искривленных наноструктурах в последнее время появились методы, сводящие задачу к анализу оператора Бельтрами-Лапласа с точечным взаимодействием на римановом многообразии [4], [5]. Также активно проводятся теоретические исследования точечных взаимодействий на искривленных многообразиях. Например, детальное описание точечных возмущений на многообразиях постоянной кривизны можно найти в [6]. В настоящей работе мы рассматриваем точечные возмущения на трехмерных многообразиях ограниченной геометрии и явно указываем способ построения аппроксимации формального выражения (1) масштабно-преобразованными операторами вида $H=H^{0}+V(x)$, где $V(x)$ - непрерывная функция с компактным носителем. Схема построения аппроксимации аналогична предложенной в [3]. Сходимость аппроксимирующих операторов будем рассматривать в равномерном резольвентном смысле.

Следует также отметить, что иной подход к аппроксимации точечных взаимодействий изложен в работе [7].

\section{2. ПРЕДВАРИТЕЛЬНЫЕ СВЕДЕНИЯ}

Здесь мы введем основные понятия и обозначения, которые будем использовать в настоящей работе. Пусть $X$ - трехмерное риманово многообразие. Всюду в дальнейшем мы будем предполагать, что $X$ - многообразие ограниченной геометрии, т.е. радиус инъективности $X$ положителен и каждая ковариантная производная риманова тензора кривизны ограничена. Более подробное описание таких многообразий можно найти в [8].

Радиус инъективности $X$ мы будем обозначать через $r_{\text {inj }}$. Кроме того, мы зафиксируем некоторое $r_{0}, 0<r_{0}<r_{\mathrm{inj}} / 3$. Под $\rho(x, y)$ мы будем понимать геодезическое расстояние между точками $x$ и $y$, через $B(a, r)$ будем обозначать шар с центром в точке $a$ радиусом $r$, т.е. $B(a, r)=\{x \in X: \rho(a, x)<r\}$. Через $L^{2}(X, \mu)$ мы обозначим пространство функций, суммируемых с квадратом на $X$ по мере $\mu$, где $\mu$ - мера Римана-Лебега на $X$. Интеграл от функции $f(x)$ по мере $\mu$ мы будем записывать как $\int_{X} f(x) d x$.

Ниже мы приведем лемму, доказанную в [9], которая является обобщением на случай многообразий ограниченной геометрии общеизвестного для евклидовых пространств факта.

ЛЕмма 1. Пусть $X$ - многообразие ограниченной геометрии размерности $\nu$, $\alpha \in \mathbb{R}, 0<\alpha<\nu$. Тогда для всех $x \in X$ и любого $r: 0<r<r_{\text {inj }}$ справедлива оценка

$$
\int_{B(a, r)} \rho(x, y)^{-\alpha} d y \leqslant c_{\alpha} r^{\nu-\alpha},
$$

где $c_{\alpha}$ - некоторая постоянная, зависящая только от $\alpha$. 
Из этой леммы совершенно очевидным образом вытекает следующая лемма.

Лемма 2. Пусть $X$ - трехмерное многообразие ограниченной геометрии, $M \subset$ $X$ - произвольное компактное множество. Тогда существует постоянная $C$, $0<C<\infty$, такая, что

$$
\int_{M \times M} \frac{d x d y}{\rho^{2}(x, y)} \leqslant C .
$$

В дальнейших рассуждениях будет использован еще один факт, доказательство которого также можно найти в [9].

ЛЕмма 3. Пусть $X$ - многообразие ограниченной геометрии размерности $\nu$, $\alpha_{1}, \alpha_{2} \in \mathbb{R}, 0<\alpha_{1}, \alpha_{2}<\nu$ и $\beta=\alpha_{1}+\alpha_{2}-\nu$. Тогда существует константа $C>0$ такая, что для всех $a \in X$, всех $r: 0<r<r_{\text {inj }} u$ всех $x, y \in B(a, r), x \neq y$,

$$
I(x, y)=\int_{B(a, r)} \rho(x, z)^{-\alpha_{1}} \rho(y, z)^{-\alpha_{2}} d z \leqslant C,
$$

если $\beta>0$.

Рассмотрим теперь некоторую точку $q \in X$. Известно (см., например, [10]), что для каждого касательного вектора $v \in T_{q} X$ существует и при том единственная геодезическая $\gamma=\gamma(t), \gamma:[0,1] \rightarrow X$, такая, что $\gamma(0)=q$ и $\gamma^{\prime}(0)=v$. Тем самым если мы рассмотрим некоторую локальную карту $V \subset X$, содержащую $q$, то каждой точке $x \in V$ можно поставить в соответствие единственную тройку чисел $\left(t, v_{1}, v_{2}\right)$, где $t=\rho(q, x)$. Полученные координаты называют полярно-геодезическими.

Учитывая сказанное, в любой окрестности рассматриваемой точки $q$, содержащейся в некоторой локальной карте, мы можем ввести отображение $\Theta_{q}(k, \cdot): x \rightarrow X$, $k \in \mathbb{R}$, которое каждой точке $x \in X$ с координатами $\left(t, v_{1}, v_{2}\right)$ ставит в соответствие точку $\Theta_{a}(k, x)$ с координатами $\left(k t, v_{1}, v_{2}\right)$. Такое отображение мы будем называть гомотетией (по аналогии с евклидовым случаем) с центром в точке $q$ и коэффициентом $k$.

Рассмотрим квантово-механический гамильтониан свободной заряженной частицы массы $M$ на многообразии $X$ :

$$
H^{0}=-\frac{\hbar}{2 M} \Delta_{\mathrm{LB}}
$$

где $\Delta_{\mathrm{LB}}$ - оператор Бельтрами-Лапласа на $X$ [11]. Далее мы будем использовать систему единиц, в которой $\hbar=1, M=1 / 2$. Отметим, что $H_{0}$ - оператор, действующий в $L^{2}(X, \mu)$. Область определения $H^{0}$ - это замыкание множества бесконечно дифференцируемых функций с компактными носителями.

\section{3. ПОСТРОЕНИЕ АППРОКСИМАЦИИ}

Рассмотрим возмущение $H^{0}$ в точке $q \in X$. Как уже отмечалось выше, формально такое возмущение задается оператором вида

$$
H_{q}=-\Delta_{\mathrm{LB}}+\lambda \delta_{q}(\cdot) .
$$


Наша цель - построить последовательность операторов с регулярными потенциалами, сходящуюся (в равномерном резольвентном смысле) к $H_{q}$.

Рассмотрим $S_{q}$ - сужение $H^{0}$ на область $D_{q}=\left\{f \in D\left(H^{0}\right): f(q)=0\right\}$. Можно показать, что $S_{q}$ - замкнутый симметрический оператор с индексами дефекта $(1,1)$, а дефектное пространство $S_{q}$ порождено функциями $x \rightarrow G^{0}(x, q ; z)$, где $G^{0}(x, y ; z)-$ функция Грина оператора $H^{0}$ (интегральное ядро резольвенты). Известно [12], что

$$
G^{0}(x, y ; z)=G_{\mathrm{reg}}^{0}(x, y ; z)+\frac{1}{4 \pi} \rho(x, y)^{-1},
$$

где $G_{\mathrm{reg}}^{0}(x, y ; z)$ - функция, непрерывная на $X \times X$.

Самосопряженные расширения оператора $S_{q}$ мы будем называть возмущениями $H^{0}$. Их можно описать, используя так называемую формулу Крейна для резольвент [13]: все расширения $S_{q}$ образуют однопараметрическое семейство операторов, резольвенты которых имеют вид

$$
R^{\alpha}(z)=R^{0}(z)-(Q(z)-\alpha)^{-1}\left\langle\overline{G^{0}(q, \cdot ; z)} \mid \cdot\right\rangle G^{0}(\cdot, q ; z),
$$

где $R^{0}(z)=\left(H^{0}-z\right)^{-1}$ - резольвента исходного оператора, а $Q(z)=G_{\mathrm{reg}}^{0}(q, q ; z)-$ $Q$-функция Крейна.

Теперь перейдем к построению последовательности операторов, аппроксимирующих возмущения $H^{0}$. Пусть $(K, \varphi)$ - произвольная локальная карта на $X$, содержащая точку $q$ и шар $B\left(q, r_{0}\right)$, и $\Theta_{q}(\varepsilon, \cdot)$ - гомотетия на $K$ с центром в точке $q$ и коэффициентом $\varepsilon$. Здесь и ниже будем предполагать, что $0 \leqslant \varepsilon \leqslant 1$.

Рассмотрим оператор

$$
H_{q}(\varepsilon)=-\Delta_{\mathrm{LB}}+\frac{\lambda(\varepsilon)}{\varepsilon^{2}} V\left(\Theta_{q}^{-1}(\varepsilon, \cdot)\right)
$$

где $\Theta_{q}^{-1}(\varepsilon, \cdot)$ - отображение, обратное к $\Theta_{q}(\varepsilon, \cdot), \lambda(\varepsilon)$ - некоторая вещественно-аналитическая в окрестности нуля функция, причем $\lambda(0)=1, V(x)$ - непрерывная функция на $X$ с компактным носителем $\operatorname{supp} V(x) \subset K$. Предположим также, что $V(x)$ неотрицательна при каждом $x \in X$.

Введем функции

$$
v(x)=(V(x))^{1 / 2}, \quad \tilde{v}(x)=v\left(\Theta_{q}^{-1}(\varepsilon, x)\right) .
$$

Тогда согласно теореме В.1 из [3]

$$
\left(H_{q}(\varepsilon)-z\right)^{-1}=R^{0}(z)-\frac{\lambda(\varepsilon)}{\varepsilon^{2}} R^{0}(z) \tilde{v}\left(1+\frac{\lambda(\varepsilon)}{\varepsilon^{2}} \tilde{v} R^{0}(z) \tilde{v}\right)^{-1} \tilde{v} R^{0}(z) .
$$

Перепишем это выражение в более удобной форме. Для этого введем масштабное преобразование на $L^{2}(X, \mu)$ по формуле

$$
\left(U_{\varepsilon} g\right)(x)=\varepsilon^{-3 / 2} g\left(\Theta_{q}^{-1}(\varepsilon, x)\right) .
$$

Тогда выражение (4) запишется в виде

$$
\left(H_{q}(\varepsilon)-z\right)^{-1}=R^{0}(z)-\varepsilon \lambda(\varepsilon) A_{\varepsilon}(z)\left(1+B_{\varepsilon}(z)\right)^{-1} C_{\varepsilon}(z),
$$



где

$$
A_{\varepsilon}(z)=\varepsilon^{-3 / 2} R^{0}(z) U_{\varepsilon} v, \quad B_{\varepsilon}(z)=\frac{\lambda(\varepsilon)}{\varepsilon^{2}} v U_{\varepsilon}^{-1} R^{0}(z) U_{\varepsilon} v, \quad C_{\varepsilon}(z)=\varepsilon^{-3 / 2} v U_{\varepsilon}^{-1} R^{0}(z)
$$

Вычислим интегральные ядра указанных операторов. Пусть $g(x) \in L^{2}(X, \mu)$, тогда

$$
\left(A_{\varepsilon}(z) g\right)(x)=\varepsilon^{-3} \int_{X} G^{0}(x, y ; z) v\left(\Theta_{q}^{-1}(\varepsilon, y)\right) g\left(\Theta_{q}^{-1}(\varepsilon, y)\right) d y .
$$

Сделаем замену переменной, положив $\tilde{y}=\Theta_{q}^{-1}(\varepsilon, y)$, тогда $y=\Theta_{q}(\varepsilon, \tilde{y})$ и $d y=\varepsilon^{3} d \tilde{y}$ (см. [10]). Окончательно получаем

$$
\left(A_{\varepsilon}(z) g\right)(x)=\int_{X} G^{0}\left(x, \Theta_{q}(\varepsilon, \tilde{y}) ; z\right) v(\tilde{y}) g(\tilde{y}) d \tilde{y} .
$$

Отсюда видно, что ядро оператора $A_{\varepsilon}(z)$ имеет вид

$$
A_{\varepsilon}(x, y ; z)=G^{0}\left(x, \Theta_{q}(\varepsilon, y) ; z\right) v(y) \text {. }
$$

Действуя аналогично, можно показать, что функции

$$
\begin{aligned}
& B_{\varepsilon}(x, y ; z)=\varepsilon \lambda(\varepsilon) v(x) G^{0}\left(\Theta_{q}(\varepsilon, x), \Theta_{q}(\varepsilon, y) ; z\right) v(y), \\
& C_{\varepsilon}(x, y ; z)=v(x) G^{0}\left(\Theta_{q}(\varepsilon, x), y ; z\right)
\end{aligned}
$$

являются интегральными ядрами операторов $B_{\varepsilon}(z)$ и $C_{\varepsilon}(z)$ соответственно.

Определим интегральные операторы $A_{0}(z), B_{0}(z), C_{0}(z)$, задав их ядра формулами

$$
\begin{aligned}
& A_{0}(x, y ; z)=G^{0}(x, q ; z) v(y), \\
& B_{0}(x, y ; z)=\frac{v(x) v(y)}{4 \pi \rho(x, y)}, \\
& C_{0}(x, y ; z)=v(x) G^{0}(q, y ; z) .
\end{aligned}
$$

Кроме того, введем еще два нужных для дальнейшего анализа оператора $D_{\varepsilon}(z)$ и $D_{0}(z)$ с ядрами

$$
D_{\varepsilon}(x, y ; z)=v(x) G_{\mathrm{reg}}^{0}\left(\Theta_{q}(\varepsilon, x), \Theta_{q}(\varepsilon, y) ; z\right) v(y)
$$

и

$$
D_{0}(x, y ; z)=v(x) G_{\mathrm{reg}}^{0}(q, q ; z) v(y)=Q(z ; q) v(x) v(y)
$$

соответственно.

Исходя из вида функций (5)-(9), леммы 2 и того факта, что функция $v(x)$ ограничена на компакте $M_{V}=\operatorname{supp} V(x)$, нетрудно убедиться в истинности следующего утверждения.

Лемма 4. Onераторь $A_{0}(z), B_{0}(z), C_{0}(z), D_{0}(z), A_{\varepsilon}(z), B_{\varepsilon}(z), C_{\varepsilon}(z), D_{\varepsilon}(z)$ являются операторами Гилъберта-Шмидта. 
Теперь сформулируем и докажем еще один факт, на который будет в существенном опираться доказательство основного результата работы.

ЛЕмма 5. Справедливы следующие асимптотические разложения, понимаемые в смысле нормы Гилъберта-Шмидта:

$$
A_{\varepsilon}(z)=A_{0}(z)+o(1), \quad C_{\varepsilon}(z)=C_{0}(z)+o(1), \quad B_{\varepsilon}(z)=B_{0}(z)+\varepsilon B_{1}(z)+o(\varepsilon),
$$

где $B_{1}(z)=\lambda^{\prime}(0) B_{0}(z)+D_{0}(z)$ nрu $\varepsilon \rightarrow+0$.

ДокАзАтЕЛЬство. Докажем первую асимптотику. Очевидно, что

$$
\left\|A_{\varepsilon}(z)-A_{0}(z)\right\|_{\mathrm{HS}}^{2} \leqslant C_{V} \int_{X} \int_{M}\left|G^{0}\left(x, \Theta_{q}(\varepsilon, y) ; z\right)-G^{0}(x, q ; z)\right|^{2} d x d y
$$

где $C_{V}=\max _{x \in M}|V(x)|$. Далее, используя формулу (2), для этого интеграла легко получить следующую оценку:

$$
\begin{aligned}
& \int_{X} \int_{M}\left|G^{0}\left(x, \Theta_{q}(\varepsilon, y) ; z\right)-G^{0}(x, q ; z)\right|^{2} d x d y \leqslant \\
& \leqslant I_{q}(\varepsilon ; z)+\frac{C_{0}(z)}{\pi} \int_{M} \int_{M}\left|\frac{1}{\rho\left(x, \Theta_{q}(\varepsilon, y)\right)}-\frac{1}{\rho(x, q)}\right| d x d y+ \\
& \quad+\frac{1}{16 \pi^{2}} \int_{M} \int_{M}\left|\frac{1}{\rho\left(x, \Theta_{q}(\varepsilon, y)\right)}-\frac{1}{\rho(x, q)}\right|^{2} d x d y
\end{aligned}
$$

где $I_{q}(\varepsilon, z) \rightarrow 0$ при $\varepsilon \rightarrow+0$, а $C_{0}(z)=\max _{M \times M}\left[G^{0}(x, y ; z)\right]$.

Найдем оценку для последнего интеграла (оценка для предпоследнего интеграла получается аналогично). Так как $M$ - компакт, найдется такой шар $B_{r}=B(q, r)$, что $M \subset B_{r}$, значит, справедлива оценка

$$
\begin{aligned}
& \int_{M} \int_{M}\left|\frac{1}{\rho\left(x, \Theta_{q}(\varepsilon, y)\right)}-\frac{1}{\rho(x, q)}\right|^{2} d x d y \leqslant \\
& \quad \leqslant \int_{B_{r}} \int_{B_{r}}\left|\frac{1}{\rho\left(x, \Theta_{q}(\varepsilon, y)\right)}-\frac{1}{\rho(x, q)}\right|^{2} d x d y .
\end{aligned}
$$

Оценку интеграла в правой части неравенства можно получить следующим образом. Разобьем область интегрирования $B_{r} \times B_{r}$ на множества $B_{r} \times\left(B_{r} \backslash B_{2 \varepsilon r}\right)$ и $B_{r} \times B_{2 \varepsilon r}$. Интеграл по первому множеству будет сходиться к нулю при $\varepsilon \rightarrow+0$ в силу регулярности подынтегральной функции. Используя леммы 1,3 , можно оценить второй интеграл некоторой функцией $F_{q}(\varepsilon, z)$, сходящейся к нулю при $\varepsilon \rightarrow+0$.

Таким образом, получаем следующую сходимость по норме Гильберта-Шмидта: $\left\|A_{\varepsilon}(z)-A_{0}(z)\right\|_{\mathrm{HS}}^{2} \rightarrow 0$ при $\varepsilon \rightarrow+0$, что и доказывает первую асимптотику.

Доказательство второго разложения полностью аналогично доказательству, приведенному выше. 
Для проверки последней асимптотики найдем асимптотику функции $B_{\varepsilon}(x, y ; z)-$ ядра оператора $B_{\varepsilon}(z)$ :

$$
\begin{aligned}
B_{\varepsilon}(x, y ; z) & =\varepsilon \lambda(\varepsilon) v(x) v(y) G^{0}\left(\Theta_{q}(\varepsilon, x), \Theta_{q}(\varepsilon, y) ; z\right)= \\
& =\varepsilon\left(1+\varepsilon \lambda^{\prime}(0)+o(\varepsilon)\right)\left(D_{\varepsilon}(x, y ; z)+\frac{1}{\varepsilon} B_{0}(x, y ; z)\right)= \\
& =B_{0}(x, y ; z)+\varepsilon\left[\lambda^{\prime}(0) B_{0}(x, y ; z)+D_{0}(x, y ; z)\right]+o(\varepsilon) .
\end{aligned}
$$

Здесь мы воспользовались тем фактом, что $\rho\left(\Theta_{q}(\varepsilon, x), \Theta_{q}(\varepsilon, y)\right) \sim \varepsilon \rho(x, y)$ при $\varepsilon \rightarrow+0$ (ввиду гладкости поля геодезических в пространствах ограниченной геометрии и непрерывности функции $\Theta_{q}(\varepsilon, x)$ по каждому из аргументов). Также нами было использовано разложение $D_{\varepsilon}(z)=D_{0}(z)+o(\varepsilon)$, которое легко проверить.

Таким образом, нами доказаны все утверждения леммы.

\section{4. ОСНОВНЫЕ РЕЗУЛЬТАТЫ}

Сформулируем основные результаты работы в виде следующей теоремы.

Tеорема. 1. Пусть $\langle v \mid \varphi\rangle=0$ для всех $\varphi \in L^{2}(X) \operatorname{ma\kappa ux,~что~} B_{0} \varphi=-\varphi$ (в частности, -1 не является собственным значением оператора $B_{0}(z)$ ). Тогда $H_{q}(\varepsilon) \rightarrow H^{0}$ при $\varepsilon \rightarrow+0$ в смысле равномерной резольвентной сходимости.

2. Пусть $\langle v \mid \varphi\rangle \neq 0$ u - - простое собственное значение $B_{0}(z), \varphi$ - соответствующая собственному значению -1 нормированная собственная функиия. Тогда $H_{q}(\varepsilon) \rightarrow H_{q}^{\alpha}$ при $\varepsilon \rightarrow+0$ в смысле равномерной резольвентной сходимости, где $\alpha=-\lambda^{\prime}(0)|\langle v \mid \varphi\rangle|^{-2}$.

ДокАЗАТЕльство. Согласно полученным выше результатам

$$
\lim _{\varepsilon \rightarrow+0} R_{\varepsilon}(z)=R_{0}(z)-A_{0}(z) F(z) C_{0}(z), \quad z \in \mathbb{C} \backslash \mathbb{R},
$$

где $F(z)=\lim _{\varepsilon \rightarrow+0}\left\{\varepsilon\left[1+B_{\varepsilon}(z)\right]^{-1}\right\}$. Вид оператора $F(z)$ зависит от спектральных свойств $B_{0}(z)$. Рассмотрим по отдельности два утверждения теоремы.

1. Пусть -1 не является собственным значением $B_{0}(z)$, тогда $-1 \in \rho\left(B_{0}(z)\right)$ в силу компактности $B_{0}(z)$. Далее, так как $\left\|B_{\varepsilon}(z)-B_{0}(z)\right\| \rightarrow 0$ при $\varepsilon \rightarrow+0$ (см. лемму 5$)$, то операторы $1+B_{\varepsilon}(z)$ ограничены для достаточно малых $\varepsilon>0$, и при этом $\left(1+B_{\varepsilon}(z)\right)^{-1} \rightarrow\left(1+B_{0}(z)\right)^{-1}$ при $\varepsilon \rightarrow+0$ в смысле равномерной сходимости. Таким образом, $F(z)=0$, что доказывает первое утверждение теоремы.

2. Прежде чем приступить к непосредственному доказательству второго случая, сформулируем следующее важное утверждение.

ЛЕмма 6. Для всех достаточно малых $z \in \mathbb{C} \backslash\{0\}$ справедливо следующее разложение:

$$
\left(1+z+B_{0}(z)\right)^{-1}=z^{-1} P+\sum_{n=0}^{\infty}(-z)^{n} T^{n+1},
$$

где $P=\langle\varphi \mid \cdot\rangle \varphi$ - проектор на собственное подпространство $B_{0}(z)$, соответствующее $-1, T-$ равномерный предел оператора $\left(1+z+B_{0}(z)\right)^{-1}(1-P)$ при $z \rightarrow 0$. 
ДокАзАтЕльСтво. Доказываемое утверждение следует из результатов раздела III.6.5 в [14], необходимо лишь доказать, что из равенства $\left(1+B_{0}(z)\right)^{2} g=0$, $g \in L^{2}(X)$, следует $\left(1+B_{0}(z)\right) g=0$.

Итак, пусть $f=\left(1+B_{0}(z)\right) g$ и $\left(1+B_{0}(z)\right) f=0$. Тогда

$$
\langle f \mid f\rangle=\left\langle\left(1+B_{0}(z)\right) g \mid f\right\rangle=\left\langle g \mid\left(1+B_{0}(z)\right) f\right\rangle=0 .
$$

Значит,

$0=\left\langle f \mid\left(1+B^{0}(z)\right) f\right\rangle=\langle f \mid f\rangle+\left\langle f \mid B_{0}(z) f\right\rangle=\int_{X}\left(B_{0}(z) f\right)(x) f(x) d x=\left\|T^{1 / 2} V^{1 / 2} f\right\|^{2}$,

где $T$ - оператор с интегральным ядром $G^{T}(x, y)=1 / 4 \pi \rho(x, y)$. Отсюда получаем $V^{1 / 2} f \equiv 0$, следовательно, $f \equiv 0$, что доказывает утверждение леммы.

Вернемся к доказательству теоремы. Используя утверждение леммы 5 , получим

$$
\begin{aligned}
\varepsilon\left(1+B_{\varepsilon}(z)\right)^{-1} & =\varepsilon\left[1+B_{0}(z)+\varepsilon B_{\varepsilon}(z)+o(\varepsilon)\right]^{-1}= \\
& =\left[1+\varepsilon\left(1+\varepsilon+B_{0}(z)\right)^{-1}\left(B_{1}(z)-1+o(1)\right)\right]^{-1} \varepsilon\left(1+\varepsilon+B_{0}(z)\right)^{-1} .
\end{aligned}
$$

Из утверждения леммы 6 вытекает

$$
\varepsilon\left(1+\varepsilon+B_{0}(z)\right)^{-1}=\varepsilon\left(\varepsilon^{-1} P+O(1)\right)=P+O(\varepsilon)=P+o(1) .
$$

Следовательно,

$$
\varepsilon\left(1+B_{\varepsilon}(z)\right)^{-1}=\left[1+P\left(B_{1}-1\right)+o(1)\right]^{-1}(P+O(\varepsilon)) .
$$

Далее воспользуемся формулой $B_{1}(z)=\lambda^{\prime}(0) B_{0}(z)+D_{0}$, где

$$
\left(D_{0} g\right)(x)=\int_{X} Q(z ; q) v(x) v(y) g(y) d y=Q(z ; q) v(x)\langle v \mid g\rangle .
$$

Итак,

$$
\begin{aligned}
1+P\left(B_{1}(z)-1\right) & =1+P\left(\lambda^{\prime}(0) B_{0}(z)+Q(z ; q) v\langle v \mid \cdot\rangle-1\right)= \\
& =1-\left(1+\lambda^{\prime}(0)\right)\langle\varphi \mid \cdot\rangle \varphi+Q(z ; q)\langle\varphi \mid v\rangle\langle v \mid \cdot\rangle \varphi .
\end{aligned}
$$

Непосредственной проверкой можно убедиться, что обратный оператор имеет вид

$$
\begin{aligned}
& \left(1+P\left(B_{1}(z)-1\right)\right)^{-1}= \\
& \quad=1-\left[\lambda^{\prime}(0)-Q(z)|\langle\varphi \mid v\rangle|^{2}\right]^{-1}\left[\left(1+\lambda^{\prime}(0)\right)\langle\varphi \mid \cdot\rangle-Q(z)\langle\varphi \mid v\rangle\langle v \mid \cdot\rangle\right] \varphi .
\end{aligned}
$$

Теперь подставим полученный результат в (11). Имеем

$$
F(z)=\left[Q(z)|\langle\varphi \mid v\rangle|^{2}-\lambda^{\prime}(0)\right]^{-1}\langle\varphi \mid \cdot\rangle \varphi .
$$

Обозначим $\lambda^{\prime}(0)|\langle\varphi \mid v\rangle|^{-2}$ через $\alpha$, получим

$$
F(z)=|\langle\varphi \mid v\rangle|^{-2}(Q(z)-\alpha)^{-1}\langle\varphi \mid \cdot\rangle \varphi .
$$


Далее получаем

$$
\left(A_{0}(z) F(z) C_{0}(z) f\right)(x)=(Q(z)-\alpha)^{-1}\left\langle\overline{G^{0}(q, y ; z)} \mid f(y)\right\rangle G^{0}(x, q ; z) .
$$

Окончательно получаем (см. (3)), что

$$
\begin{aligned}
\lim _{\varepsilon \rightarrow+0} R_{\varepsilon}(z) & =R_{0}(z)-A_{0}(z) F(z) C_{0}(z)= \\
& =R_{0}(z)-(Q(z)-\alpha)^{-1}\left\langle\overline{G^{0}(q, y ; z)} \mid f(y)\right\rangle G^{0}(x, q ; z)=R_{\alpha}(z),
\end{aligned}
$$

где $\alpha=\lambda^{\prime}(0)|\langle\varphi \mid v\rangle|^{-2}$. Что и требовалось доказать.

Благодарности. Работа частично поддержана РФФИ (грант № 09-01-90410).

\section{Список литературы}

[1] R. de L. Kronig, W. G. Penney, Proc. R. Soc. Lond. Ser. A, 130:814 (1931), 499-513.

[2] Ф. А. Березин, Л. Д. Фаддеев, ДАН СССР, 137 (1961), 1011-1014.

[3] С. Альбеверио, Ф. Гестези, Р. Хеэг-Крон, Х. Холден, Решаемые модели в квантовой механике, Мир, М., 1991.

[4] A. V. Chaplik, L. I. Magarill, D. A. Romanov, Phys. B, 249-251 (1998), 377-382.

[5] V. V. Gritsev, Yu. A. Kurochkin, Phys. Rev. B, 64:3 (2001), 035308.

[6] I. V. Krive, S. Naftulin, A.S. Rozhavsky, Ann. Phys., 232:2 (1994), 225-242.

[7] Л. П. Нижник, Укр. матем. журн., 49:11 (1997), 1557-1560.

[8] M. A. Shubin, "Spectral theory of elliptic operators on noncompact manifolds", Méthodes semi-classiques, V. 1, Astérisque, 207, ed. D. Robert, Soc. Mat. France, Paris, 1992, 35-108.

[9] J. Brüning, V. Geyler, K. Pankrashkin, Ann. Henri Poincaré, 8:4 (2007), 781-816.

[10] M. Berger, A Panoramic View of Riemannian Geometry, Springer, Berlin, 2003.

[11] C. Groshe, F. Steiner, Handbook of Feynman Path Integrals, Springer Tracts Modern Phys., 145, Springer, Berlin, 1998.

[12] J. Brüning, V. Geyler, V. Pankrashkin, J. Math. Phys, 46:11 (2005), 113508.

[13] Б. С. Павлов, УМН, 42:6(258) (1987), 99-131.

[14] Т. Като, Теория возмущений линейных операторов, Мир, М., 1972.

Поступила в редакцию 13.12.2007, после доработки 22.03.2008 